\title{
Fault-Tolerant SemiFast Implementations of Atomic Read/Write Registers
}

\author{
Chryssis Georgiou \\ University of Cyprus, Cyprus \\ chryssis@cs.ucy.ac.cy
}

\author{
Nicolas C. Nicolaou \\ University of Connecticut, USA \\ nicolas@engr.uconn.edu
}

\author{
Alexander A. Shvartsman \\ University of Connecticut and \\ MIT, USA \\ aas@engr.uconn.edu
}

\begin{abstract}
This paper investigates time-efficient implementations of atomic read-write registers in message-passing systems where the number of readers can be unbounded. In particular we study the case of a single writer, multiple readers, and $S$ servers, such that the writer, any subset of the readers, and up to $t$ servers may crash. A recent result of Dutta et al. [3] shows how to obtain fast implementations in which both reads and writes complete in one communication round-trip, under the constraint that the number of readers is less than $\frac{S}{t}-2$, where $t<\frac{S}{2}$. In that same paper the authors pose a question of whether it is possible to relax the bound on readers, and at what cost, if semifast implementations are considered, i.e., implementations that have fast reads or fast writes.

This paper provides an answer to this question. It is shown that one can obtain implementations where all writes are fast, i.e., involving a single round-trip communication, and where reads complete in one to two communication rounds under the assumption that no more than $t<\frac{S}{2}$ servers crash. Simulated scenarios included in this paper indicate that only a small fraction of reads require a second communication round. Interestingly the correctness of the implementation does not depend on the number of concurrent readers in the system. The solution is obtained with the help of non-unique virtual ids assigned to each reader, where the readers sharing a virtual id form a virtual node. For the proposed definition of semifast implementations it is shown that implementations satisfying certain assumptions are semifast if and only if the number of virtual ids in the system is less than $\frac{S}{t}-2$. This result is proved to be tight in terms of the required communication. It is shown that only a single complete two-round read operation may be necessary for each write operation. It is furthermore shown that no semifast implementation exists for the multi-reader, multi-writer model.
\end{abstract}

*This work is supported in part by the NSF Grants 9988304 , 0121277 , and 0311368 .

${ }^{\dagger}$ The work of this author is supported in part by research funds at the University of Cyprus.

Permission to make digital or hard copies of all or part of this work for personal or classroom use is granted without fee provided that copies are not made or distributed for profit or commercial advantage and that copies bear this notice and the full citation on the first page. To copy otherwise, to republish, to post on servers or to redistribute to lists, requires prior specific permission and/or a fee.

SPAA'06, July 30-August 2, 2006, Cambridge, Massachusetts, USA. Copyright 2006 ACM 1-59593-262-3/06/0007 ...\$5.00.

\section{Categories and Subject Descriptors}

F.1.2 [Computation by Abstract Devices]: Modes of Computation-Parallelism and concurrency; D.4.5 [Operating Systems]: Reliability-Fault-tolerance; F.2.m [Analysis of Algorithms and Problem Complexity]: Miscellaneous

\section{General Terms}

Algorithms, Reliability, Theory

\section{Keywords}

Fault-tolerance, Distributed algorithms, Atomicity, Read/Write registers, Communication rounds

\section{INTRODUCTION}

Atomic (linearizable) read/write memory is one of the fundamental abstractions in distributed computing. Fault-tolerant implementations of atomic objects in message-passing systems allow processes to share information with precise consistency guarantees in the presence of asynchrony and failures. A seminal implementation of atomic memory of Attiya et al. [1] gives a single-writer, multiple reader (SWMR) solution where each data object is replicated at $n$ message-passing nodes. In this solution memory access operations are guaranteed to terminate as long as the number of crashed nodes is less than $n / 2$, i.e., the solution tolerates crashes of any minority of the nodes. The write protocol involves a single round-trip communication stage, while the read protocol involves two round-trip stages, where the second stage essentially performs the write of the value obtained in the first stage. Following this development, a folklore belief developed that in messaging-passing atomic memory implementations "atomic reads must write". However, recent work by Dutta et al. [3] established that if the number of readers is appropriately constrained with respect to the number of replicas, then single communication round implementations of reads are possible. Such an implementation given in [3] is called fast. Furthermore it was shown that any implementation with a larger set of readers cannot have only the single round-trip reads. Thus when the number of readers can be large, it is interesting to consider semifast implementations where the writes involve a single communication round and where the reads may involve one or two rounds with the goal of having as many as possible single round reads.

Background Details. The implementation of atomic SWMR objects in [1] uses value-timestamp pairs to impose a partial order on read and write operations. To perform a write operation, the writer increments its local timestamp and sends a message with the value-timestamp pair to all processes. When a majority of pro- 
cesses reply, the write completes. The process performing a read operation sends out queries and waits for a majority of the processes to reply with their value-timestamp pairs. When a majority of the processes replies, the reader finds the highest timestamp and sends the pair consisting of this timestamp and its associated value to all processes. The read completes when the reader receives responses from a majority of processes. Although the value of the read is established after the first communication round, skipping the second round may lead to violations of atomicity when reads are concurrent with a write.

Subsequent works extended the approach in [1] to multiple writers, each involving a two round-trip communication protocol, and using quorums of replicas instead of majorities $[8,4]$. A fully dynamic atomic memory implementation using reconfigurable quorums is given in [7], where the sets of object replicas can arbitrarily change over time as processes join and leave the system. When the set of replicas is not being reconfigured, the read and write protocols involve two communication rounds. Retargetting this work to ad-hoc mobile networks, Dolev et al. [2] formulated the GeoQuorums approach where replicas are implemented by stationary focal points that in turn are implemented by mobile nodes. Interestingly, in this work some reads involve a single communication round when it is confirmed that the previous write of the value obtained by the read has already completed.

The implementation of atomic SWMR objects in [3] assumes asynchronous message-passing systems with reliable channels. Here read and write operations are fast, i.e., involve a single communication round, but under the constraint that $R<\frac{S}{t}-2$, where $S$ is the number of servers maintaining object replicas, $R$ is the number of readers, such that the writer, any subset of readers, and up to $t$ servers may crash. Note that for any number $t \geq 1$ of failures the number of readers must be strictly less than the number of servers, and the number of readers is inversely proportional to the number of server failures. A fast implementation cannot exist in the case of multiple readers and multiple writers. For example, it is shown that in the setting where 2 writers and 2 readers exist in the system and $t=1$, atomicity can be violated.

Our Contributions. Our goal is to develop atomic memory algorithms where a large number of read and write operations are fast, i.e., involving a single communication round. In particular, we want to remove constraints on the number of readers while preserving atomicity. We say that an atomic SWMR implementation is semifast if write operations take a single communication round and where read operations take one or two rounds. We show that one can obtain semifast implementations with unbounded number of readers, where in many cases reads take a single round. Our approach is based on forming groups of processes where each group is given a unique virtual identifier. The algorithm is patterned after the general scheme of the algorithm in [3]. We show that for each write operation at most one complete read operation returning the written value may need to perform a second communication round. Furthermore, our implementation enables non-trivial executions where both reads and writes are fast, i.e., involve a single communication round. We also provide simulation results for our algorithm, and we consider semifast implementations for multiple writers. More broadly, our contributions are as follows.

1. We define the notion of a semifast implementation which specifies which atomic reads are required to perform a second communication round. In particular, for each write operation, only one complete read operation is allowed to perform two communication rounds.

2. We provide a semifast implementation of an atomic $\mathrm{read} / \mathrm{write}$ object that supports arbitrarily many readers. To accommodate arbitrarily many readers, we introduce the notion of virtual identifiers and allow multiple readers to share the same virtual identifier, thus forming groups of nodes that we call virtual nodes. We base the determination of the proper return value on the cardinality of the set of virtual nodes maintained by the servers (this is similar to the algorithm in [3] that uses the cardinality of the set of the readers maintained by the servers to determine the return value) . We prove the correctness (atomicity) of the new implementation. We note that our implementation is not a straightforward extension of [3]. The introduction of virtual nodes raises new challenges such as ensuring consistency within groups so that atomicity is not violated by processes sharing the same virtual id, and proving the resulting implementation correct.

3. We consider two families of algorithms, one that does not use reader grouping mechanisms, the other that assumes grouping mechanisms such as our algorithm. For both we show that there is no semifast atomic implementation if $\frac{S}{t}-2$ or more virtual identifiers (groups) exist in the system. Additionally it is shown that any semifast algorithm must inform no less than $3 t+1$ server processes during a second communication round.

4. We show that there does not exist semifast atomic implementations for multiple writers and multiple readers, even for $t=1$.

5. We simulated our SWMR implementation and we present preliminary results demonstrating that only a small fraction of read operations need to perform a second communication round. Specifically, under reasonable execution conditions in our simulations no more than $10 \%$ of the read operations required a second round.

Paper Organization. In Section 2 we present our model and definitions. In Section 3 we describe our implementation and prove its correctness. In Section 5 we show the necessary properties that an implementation of an atomic register must possess in order to be semifast. In Section 6 we show that no semifast MWMR implementation is possible. Section 7 contains simulation results. Due to space limitations, some proofs are omitted, and they can be found in [5].

\section{MODEL AND DEFINITIONS}

We consider the single writer, multiple reader (SWMR) model, where a distinguished process $w$ is the writer, the set of $R$ readers are processes with unique ids from the set $\mathcal{R}=\left\{r_{1}, \ldots, r_{R}\right\}$, and where the object replicas are maintained by the set of $S$ servers with unique ids from the set $\mathcal{S}=\left\{s_{1}, \ldots, s_{S}\right\}$ such that at most $t$ servers can crash. A virtual node is an abstract entity that consists of a group of reader processes. Each virtual node has a unique identifier from the set $\mathcal{V}=\left\{\nu_{1}, \ldots, \nu_{V}\right\}$, where $V<\frac{S}{t}-2$. A reader $r_{i}$ that is a member of a virtual node $\nu_{j}$ maintains its own identifier $r_{i}$ and its virtual identifier $\nu\left(r_{i}\right)=\nu_{j}$; we identify such process by the pair $\left\langle r_{i}, \nu_{j}\right\rangle$. The processes that share the same virtual identifier are called siblings. We assume that some external service is used to create virtual nodes by assigning virtual ids to reader processes. (Note that when $V=R$ and when each virtual node consists of a single unique reader, then our model is essentially that of [3].)

Each process $p$ is associated with an application. The application asks the process to invoke an operation and the process responds to the application with the result. We assume a reliable channel be- 
tween any two processes and that the messages carry a source and a destination field. The state of all channels is represented by the set mset that contains all messages sent but not yet delivered, such messages are said to be in transit. We refer to the messages that intend to write a new value to the atomic register as WRITE messages, and we call the messages that request the value of the register as READ messages. The messages used to propagate information within the system are called INFORM messages.

An algorithm $A$ is a collection of automata, where $A_{p}$ is the automaton assigned to the process $p$ with an initial state Init. Computation of $A$ proceeds in steps where each step denotes actions of a single process. In particular each step is described by an ordered tuple $\langle s t, p, m I n, i n v, m O u t, r e s\rangle$ where $s t$ is the state of the system (with $s t_{p}$ denoting the state component of process $p$ ) and includes the set of messages mset and the state of each process in the system; $p$ is the process id, $m I n$ the messages received by the process $p$ in that step, inv the invocation submitted to process $p$ by the application, $\mathrm{mOut}$ the output messages of process $p$, and res is the response of the process to the application in that step. When $i n v=\perp$ there is no invocation at that step and when $r e s=\perp$ there is no response to the application. When $m I n=\emptyset$ or $m O$ ut $=\emptyset$ then there are no messages to be received or to be sent out in that step respectively. In every step a process $p$ acts as follows, where $s t^{\prime}$ is the resulting state (the state components of all other processes are unchanged in $s t^{\prime}$ ): (1) it sets $s t^{\prime} . m s e t$ to st.mset $-m I n,(2)$ inputs $m I n, i n v$, and its current state $s t_{p}$ to $A_{p}$, which outputs a new state $s t_{p}^{\prime}$, the messages $m O u t$ to be sent, and the response res to the last invoked operation, and (3) adopts the state $s t_{p}^{\prime}$ as its new state, sets $s t^{\prime}$.mset to st.mset $\cup$ mOut, and responds with res to the application. A process $p$ performs an invocation step if the invocation inv $\neq \perp$, a response step when $r e s \neq \perp$, and a communication step if $m O u t \neq \emptyset$ and both inv $=\perp$ and res $=\perp$.

An execution fragment $\varphi$ of an algorithm $A$ is a finite or infinite sequence of steps $\sigma_{0}, \sigma_{1}, \ldots, \sigma_{r}, \ldots$ of $A$. An execution fragment is called an execution of $A$ if it begins with the step $\sigma_{0}=\langle s 0, *, *, *, *, *\rangle$ where $s 0$ is the initial state of the system and $s 0$.mset $=\emptyset$, and for each process $p, s 0_{p}=$ Init. Executions are denoted by the symbol $\xi$. A finite execution fragment $\varphi$ is a finite prefix of some execution. We say that an execution fragment $\varphi$ extends some finite execution fragment $\varphi^{\prime}$ if the first step in $\varphi$ is $\sigma_{f}=\left\langle s t^{\prime}, *, *, *, *, *\right\rangle$, the last step of $\varphi^{\prime}$ is $\sigma_{\ell}=\langle s t, *, *, *, *, *\rangle$, such that $s t^{\prime}$ is the state that immediately results from $\sigma_{\ell}$.

A process can crash during any step of an execution. Following a crash the process does not perform any steps. A process is considered to be faulty in execution $\xi$ if it crashes in $\xi$; otherwise the process is correct.

Atomicity. Our goal is to implement a read/write atomic object in a message passing system by replicating the value of the object among the servers in the system. Each replica consists of a value $v$, initially $\perp$, and an associated timestamp $t s$, initially 0 . A read or a write operation consists of an invocation step and a matching response step. An operation is incomplete in an execution, if the operation's invocation step does not have a matching response step; otherwise the operation is complete. We assume that application executions are well-formed in that it invokes one operation at a time: it waits for a response before invoking another operation.

In an execution we say that a (read or write) operation $\pi_{1}$ precedes another operation $\pi_{2}$ (or $\pi_{2}$ succeeds $\pi_{1}$ ), if the response step for $\pi_{1}$ precedes the invocation step of $\pi_{2}$. Two operations are concurrent if neither precedes the other.
Correctness of an implementation of an atomic object is defined in terms of the termination and atomicity properties. The termination property requires that any operation invoked by a correct process eventually completes. Atomicity is defined as follows [6]: Consider the set $\Pi$ of all complete operations in any well-formed execution. Then there exists an irreflexive partial ordering $\prec$ on operations in $\Pi$, satisfying the following: (1) For any operation $\pi \in \Pi$, there are finitely many operations $\pi^{\prime}$ such that $\pi^{\prime} \prec \pi$. (2) If operation $\pi_{1}$ precedes the operation $\pi_{2}$ in $\Pi$, then it cannot be the case that $\pi_{2} \prec \pi_{1}$. (3) If $\pi$ is a write operation and $\pi^{\prime}$ is any operation in $\Pi$, then either $\pi \prec \pi^{\prime}$ or $\pi^{\prime} \prec \pi$. (4) The value returned by a read operation is the value written by the last preceding write operation according to $\prec$ (or $\perp$ if there is no such write).

Semifast implementations. We say that a read or write operation $\pi$ is fast if it completes in one communication round. Let denote by $\operatorname{inv}(\pi)$ the invocation of operation $\pi$ requested at process $p$ and let $\operatorname{ret}(\pi)$ denote the response of process $p$ for operation $\pi$. We then define a communication round as follows:

DEFINITION 2.1. A process $p$ performs a communication round during operation $\pi$ in an execution if all of the following hold:

(1) $p$ sends the messages $m \in m O u t$, during an invocation step where inv $=i n v(\pi)$ or a communication step during $\pi$, to a subset of processes,

(2) any process $p^{\prime}$ that receives $m \in m I n$ during a step $\sigma$, replies to $p$ with a message $m^{\prime} \in m$ Out within the same step, ${ }^{1}$

(3) when $p$ receives at least one messages $m^{\prime} \in m I n$, it either performs a response step with res $=\operatorname{ret}(\pi)$ or inserts a set of messages in mOut and performs a communication step.

When process $p$ decides to respond to the application within $\pi$ in (3) above during the first communication round in $\pi$, then we say that operation $\pi$ is fast. An implementation is fast if both reads and writes are fast in every execution.

A semifast atomic implementation, as suggested in [3], is the implementation that either has all reads that are fast or all writes that are fast. Here we formalize the notion of semifast implementations. Let $w_{k}$ be the $k^{t h}(k \geq 1)$ write operation by the sole writer and let $v a l_{k}$ be the value written to the register. Let $\prec$ be the partial order defined on any (atomic) execution as given earlier. We use the reading-function $\mathfrak{R}(\rho)$ as defined in [9] to specify the (always unique) write operation that wrote the value returned by read $\rho$.

DEFINITION 2.2. An SWMR implementation I is semifast if the following are satisfied:

(1) In any execution $\xi$ of $I$, every write operation $w_{k}, k \geq 1$, is fast.

(2) In any execution $\xi$ of I, any complete read operation performs one or two communication rounds between the invocation and response.

(3) For any execution $\xi$ of $I$, if a two-round read operation $\rho_{1}$ returns the value ret $\left(\rho_{1}\right)=$ val $_{k}$ and $\mathfrak{R}\left(\rho_{1}\right)=w_{k}$, then any read operation $\rho_{2}$, where $\rho_{1} \prec \rho_{2}$ or $\rho_{2} \prec \rho_{1}$, and $\operatorname{ret}\left(\rho_{2}\right)=\operatorname{val} l_{k}$ and $\mathfrak{R}\left(\rho_{2}\right)=w_{k}$, must be fast.

(4) There exists an execution $\xi$ of I containing a write operation $w_{k}$ and a set of read operations $\mathcal{F}$ such that $\forall \rho \in \mathcal{F}, \mathfrak{R}(\rho)=w_{k}$ and $\rho$ is fast.

${ }^{1}$ Notice that process $p^{\prime}$ replies to $m$ either at the same step $\sigma$ or during a subsequent step $\sigma^{\prime}$, if $p^{\prime}$ does not receive any messages between $\sigma$ and (inclusively) $\sigma^{\prime}$. Intuitively this property is used to forbid processes to wait for other messages before replying to $m$. 
We make the following observations having the above definition in mind. Given that any subset of the readers and the writer may fail, in order to guarantee termination, no operation can wait for replies from any reader or writer processes. Since we require that the writes are fast, the servers cannot wait for any messages before replying to a WRITE message. Read operations on the other hand are allowed to perform two communication rounds. Two-round reads can have one of the two forms: (i) the reader process may contact the servers twice, (ii) the reader may send messages to the servers during the first round, the servers perform a communication step and contact other servers in the second round and then reply to the reader ending the first round. If the servers are responsible for the second communication round, then it may be the case that all read operations need two rounds to complete, violating semifast properties (3) and (4). Worse yet, a server may fail during its second round preventing an operation from completing. Hence both communication rounds must be performed by the reader when it decides it is necessary to do so according to the information gathered during the first round. Thus in the sequel we assume that the servers in the semifast implementation, upon receiving a READ or INFORM message, cannot wait for messages from any other process before replying. (Alternatively we can construct executions of a semifast implementation, where only the READ, WRITE and INFORM messages from the invoking processes to the servers and the replies from the servers are delivered. All the other messages remain in transit.)

\section{IMPLEMENTATION SF}

We now present a semifast implementation, called SF, in which there is one writer and arbitrarily many readers. We assume that the number $V$ of unique virtual ids is such that $V<\frac{S}{t}-2$ (we show in Section 5 that semifast implementations are possible iff $\left.V<\frac{S}{t}-2\right)$. We now describe our implementation presented in pseudocode in Figure 1. Recall that each replica consists of a value and its timestamp. For simplicity we give the algorithm that returns only the timestamps; then we describe a straightforward modification that returns a value along with each timestamp.

Writer. During a write operation, the writer $w$ sends a write message consisting of the current timestamp and the value to be written to all servers. Since $t$ of the servers might be faulty, $w$ waits for responses from only $S-t$ servers. Upon receipt of all the expected acknowledgments the writer increases its timestamp and completes the operation. The timestamps impose a natural order on the writes since there is only one writer.

Server. The servers maintain the replicas of the object. The state of a server includes the following: (1) ts the greatest timestamp received any server, (2) the set seen where the server records the virtual ids of the readers that read the latest timestamp of the server, (3) the counter array in order to distinguish new from old messages from each process (needed because of asynchrony), and (4) the variable postit used by readers to inform, if necessary, other readers about the timestamp they are about to return.

We now describe the operation of a server $s_{i}$ when it receives a message (msgType, ts ${ }^{\prime}$, rCounter $^{\prime}$, vid) from a non-server process $p_{j}$. Upon receipt of this message, server $s_{i}$ updates its timestamp $t s$ if $t s^{\prime}>t s$ and initializes its seen set to $\left\{\nu\left(p_{j}\right)\right\}$, the virtual id of $p_{j}$. Otherwise, if $t s^{\prime}<t s, s_{i}$ sets its seen set to be equal to seen $\cup\left\{\nu\left(p_{j}\right)\right\}$ declaring that $p_{j}$ perceived $s_{i}$ 's timestamp. This is a departure from the algorithm in [3]: we record the virtual identifier of $p_{j}$, using its unique identifier only for message exchange. By doing so we manage to keep $\mid$ seen $\mid<\frac{S}{t}-2$ (required for cor- rectness) without having to bound the number of readers. Server $s_{i}$ then sends a reply to $p_{j}$ acknowledging the transaction. If a READ, WRITE or INFORM message is received then the reply is a READACK, WRITEACK, or an INFORMACK, respectively. An INFORM message denotes that the process $p_{j}$ wants to inform the rest of the reader processes about the timestamp it is about to return. Accordingly, before replying to an INFORM message, $s_{i}$ updates its postit value. To ensure that the value enclosed in the INFORM message is not an already-returned timestamp, $s_{i}$ compares the received timestamp with its postit. If the postit value is greater, it must be the case that another reader already returned a newer timestamp than the one in the message and so updating the postit with an older timestamp may violate atomicity; otherwise postit is updated. Along with any reply, $s_{i}$ encloses its timestamp $t s$, its seen set, its counter, and the value of postit.

Reader. The actions of a reader node with id $r_{i}$ and virtual id $\nu\left(r_{i}\right)=\nu_{j}$ are as follows. When $r_{i}$ invokes a read operation, it sends messages to all servers and waits for $S-t$ responses. Each of these responses is of the form (READACK, $t s^{\prime}$, seen, $r$ Counter, postit). Upon collecting these messages, $r_{i}$ checks $r$ Counter to distinguish new messages from the stale messages (due to asynchrony), and then records the maximum timestamp $\max T S=t s^{\prime}$ and the maximum postit $\operatorname{maxPS}=$ postit value contained among the received messages. Based on the received information, reader $r_{i}$ computes the set of messages that contained the maximum timestamp ( $\max T S m s g$ ). Then the following predicate is used to decide the return value: we check if there is a subset $M S \subseteq \max T S m s g$ such that its cardinality $|M S| \geq S-\alpha t$, for some $\alpha \in[1, V+1]$ and the cardinality of the intersection of the messages in $M S$ is $\mid \cap_{m \in M S}$ m.seen $\mid \geq \alpha$, then we return $\max T S$. The predicate can be interpreted as "enough processes have seen the $\max T S$ that we received".

In order to visualize the idea behind the predicate consider an finite execution fragment $\varphi_{1}$ where the writer $w$ performs a complete write operation $\omega_{1}$ which receives replies from $\left|S_{w(1)}\right|=$ $S-t$ servers. We extend $\varphi_{1}$ by a complete read operation $\rho_{1}$ which misses $t$ servers from those that responded to $\omega_{1}$, such that $\left|M S_{1}\right|=\left|S_{w(1)} \cap S_{1}\right|=S-2 t$ where $S_{1}$ is the set of $S-t$ servers that responded to $\rho_{1}$. According to atomicity, the read operation $\rho_{1}$ returns $T S_{1}=\max T S$. Consider now another execution $\varphi_{2}$ where the write operation $\omega_{1}$ is incomplete and receives replies from exactly $\left|S_{w(1)}\right|=S-2 t$ servers. We extend $\varphi_{2}$ with a read operation $\rho_{1}$ from $r_{i}$ which receives replies from $\left|S_{1}\right|=S-t$ servers including the servers in $S_{w(1)}$. So $\left|M S_{1}\right|=\left|S_{w(1)} \cap S_{1}\right|=S-2 t$ and thus the read $\rho_{1}$ cannot distinguish execution $\varphi_{2}$ from $\varphi_{1}$. Hence, by atomicity, $\rho_{1}$ returns $T S_{1}=\max T S$ in $\varphi_{2}$ as well. By extending $\varphi_{2}$ even further by a second read operation $\rho_{2}$ from $r_{j}$ we might get into the situation where $\left|M S_{2}\right|=\left|S_{w(1)} \cap S_{2}\right|=S-3 t$, where $\left|S_{2}\right|=S-t$ the servers that responded to $\rho_{2}$. But in order to preserve atomicity the reader $r_{j}$ must also return $T S_{2}=\max T S$. This scenario can be easily generalized for more than two read operations and so the predicate in line 23 of the algorithm in Figure 1 arise to preserve atomicity between the different read operations.

Note here that the above result is true if the unique ids of the readers are recorded in the seen set. If we record the virtual ids of $r_{i}$ and $r_{j}$ (as it is done in our implementation) we only get the same result if the two readers are not siblings. In different case, namely where $\nu\left(r_{i}\right)=\nu\left(r_{j}\right)=\nu_{k}$, the seen set witnessed by both $\rho_{1}$ and $\rho_{2}$ in $\xi_{2}$ could be $\mid \cap_{m \in M S_{1}}$ m.seen $|=| \cap_{m \in M S_{2}}$ m.seen $\mid=$ $\left|\left\{w, \nu_{k}\right\}\right|=2$. If so the predicate would not hold for $\rho_{2}$, returning $\max T S-1$ and violating atomicity. 


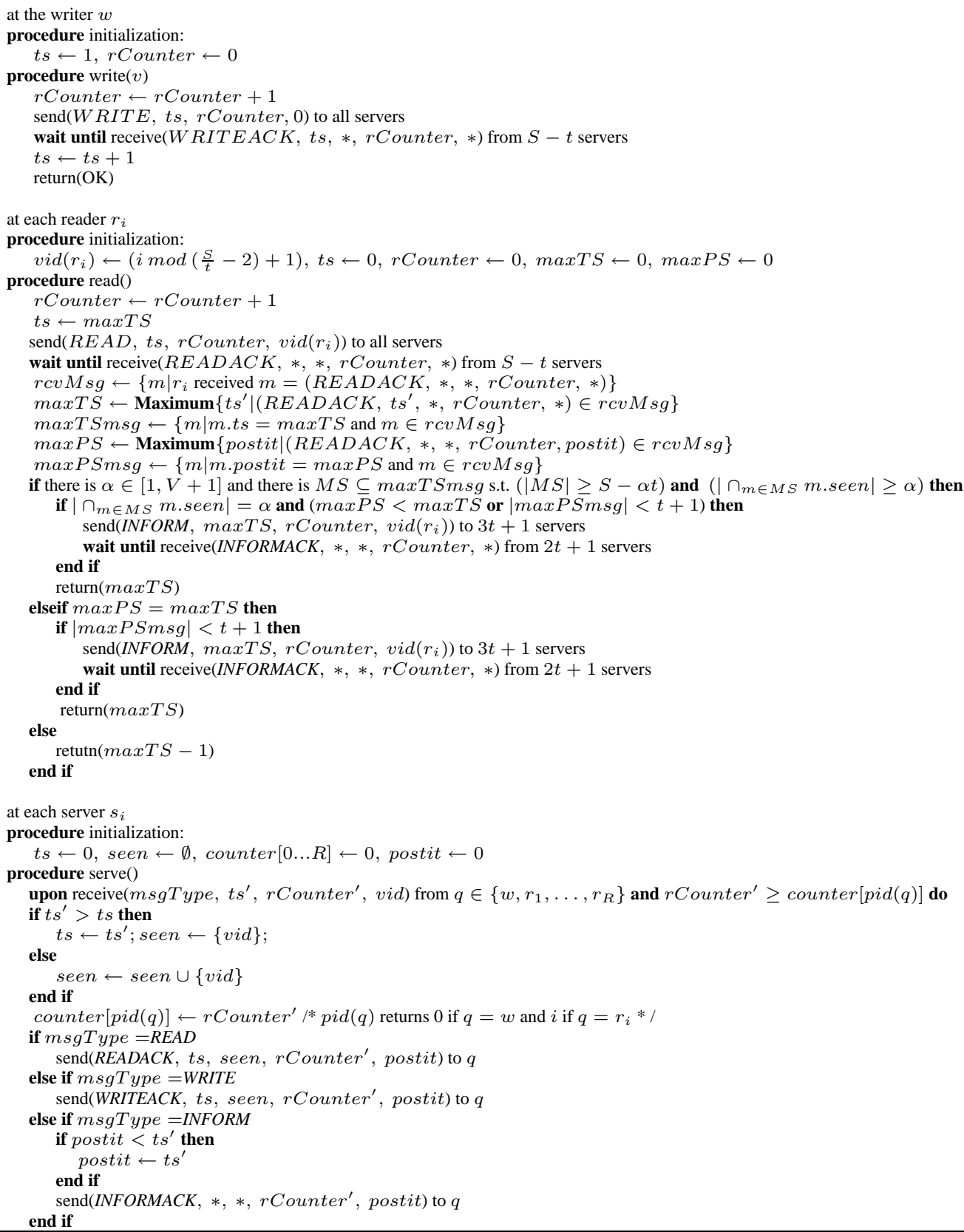

Figure 1: Implementation SF 
A second communication round is necessary when $r_{i}$ satisfies the predicate such that $\mid \cap_{m \in M S}$ m.seen $\mid=\alpha$. During the second communication round, $r_{i}$ informs $3 t+1$ servers about the timestamp it is about to return. Since $t$ servers might be faulty, $r_{i}$ completes as soon as it receives $2 t+1$ acknowledgments and returns $\max T S$.

In the case where the predicate is false, reader $r_{i}$ checks if there was any postit equal to $\max T S$ observed, as advertised within the received messages. If so, then some reader (previously or concurrently with $r_{i}$ ) returned or is about to return $\max T S$. If $r_{i}$ receives more than $t+1$ messages containing that postit, it returns $\max T S$ without performing a second communication round; otherwise a second communication round is required by $r_{i}$ to ensure that any subsequent reader will receive the same postit. If neither postit equals $\max T S$, then $r_{i}$ returns $\max T S-1$ in one communication round.

Remark: By the above implementation, if all readers form one virtual node $(V=1)$, then a read operation $\rho$ will return $\max T S$ only when it receives at least $S-2 t$ replies which contain $\max T S$. But this implies that the write operation which wrote $\max T S$ must be either completed or requires at most $t$ more replies to complete. Consequently in order to achieve efficiency, it is important to study the division of the reader processes among the virtual nodes. This is left as an open question.

Returning values with timestamps. A slight modification needs to be applied to the algorithm to associate returned timestamps with values. To do this the writer attaches two values to the timestamp in each write operation: (1) the current value to be written, and (2) the value written by the immediately preceding write operation (for the first write this is $\perp$ ). The reader receives the timestamp with its two associated values and if it decides (as before) to return $\max T S$, then it returns the current value attached to $\max T S$. If the reader decides to return $\max T S-1$, then it returns the second value (that of the preceding write).

We now give the correctness of algorithm SF.

THEOREM 3.1. Algorithm SF implements a semifast atomic SWMR read/write register.

Proof. (Sketch.) The proof is done in two parts. We first show that SF implements an atomic read/write register in the SWMR model by showing that in any execution the atomicity properties are not violated (see Section 4). Then we show that SF is a semifast implementation by showing that the requirements of Definition 2.2 are met.

\section{CORRECTNESS OF SF}

Since the correctness of our implementation depends mainly on the timestamps written and returned, we reduce the properties of the atomicity presented in Section 2, to the following: (1) If a read operation returns, it returns a non-negative integer, (2) if a read $\rho$ is complete and succeeds some write $(k)$, then $\rho$ returns $\ell$ such that $\ell \geq k$, (3) if a read $\rho$ returns $k(k \geq 1)$, then write $(k)$ either precedes $\rho$ or is concurrent with $\rho$, (4) if some read $\rho_{1}$ returns $k(k \geq 0)$ and a read $\rho_{2}$ that succeeds $\rho_{1}$ returns $\ell$, then $\ell \geq k$. We will show that implementation SF preserves each and every of the above conditions in any given execution.

Before proceeding to the proof we first introduce some notation we use throughout this section. Each read operation is denoted by $\rho_{i}$. For each read operation $\rho_{i}$, let $S_{i}$ denote the set of servers that received messages from $\rho_{i}$ and replied to those messages. For the writer we denote the set of servers that received messages from the $k^{t h}$ write operation as $S_{w(k)}$. Furthermore let $\operatorname{Max} S_{i}$ be the set of servers that replied with the maximum timestamp to $\rho_{i}$, and therefore $\operatorname{Max} S_{i} \subseteq S_{i}$. The set of messages received from $\rho_{i}$ containing the maximum timestamp and sent by the servers in $\operatorname{Max}_{i}$, is represented by $M S_{i}$. The maximum timestamp received by the read $\rho_{i}$ is represented as $T S_{i}$. If a read operation $\rho_{i}$ performs a second communication round, then we denote as $N S_{i}$ to be the set of servers that received the messages from the second communication round of $\rho_{i}$ and replied to those messages. We say that a read operation $\rho_{i}$ is invoked by the reader $\left\langle r_{j}, \nu_{k}\right\rangle$, where $r_{j}$ is the identifier and $\nu_{k}$ the virtual identifier of the reader. Lastly for a process $p$ we denote as $t s_{p}$ the value of the timestamp of $p$ and as postit the value of the postit variable at $p$.

We begin with a lemma that plays a significant role in the correctness of our implementation. The lemma follows from the fact that no more than $t$ servers might fail and that the communication channels are reliable.

LEMMA 4.1. Let two readers $\left\langle r_{i}, \nu_{*}\right\rangle$ and $\left\langle r_{j}, \nu_{*}\right\rangle$ perform subsequent reads $\rho_{1}$ and $\rho_{2}$, respectively. Then, for any execution $\xi$ of $S F,|| \operatorname{Max} S_{1}|-| \operatorname{Max} S_{2}|| \leq t$.

The proofs of the first and third atomicity conditions (as given above) are omitted because of their triviality.

LEMMA 4.2. In any execution $\xi$ of $S F$, if a server $s_{i}$ sets its timestamp $t_{s_{i}}$ to $x$ at step $\sigma$, then, given any step $\sigma^{\prime}$ of $\xi$ such that $\sigma<\sigma^{\prime}$ and $t s_{s_{i}}=y$, we have that $y>x$.

PROOF. This can be ensured by line 44 of Figure 1 .

We now show the monotonicity of the postits for any server.

LEMMA 4.3. In any execution $\xi$ of $S F$, if a server $s_{i}$ sets its postit $_{s_{i}}$ to $x$ at a step $\sigma$, then, given any step $\sigma^{\prime}$ of $\xi$ such that $\sigma<\sigma^{\prime}$ and postit s $_{i}=y$, we have that $y>x$.

PROOF. This can be ensured by line 55 of Figure 1.

The following lemma ensures that if a postit $=x$ is introduced to the system, then there exists a maximum timestamp ts in the system such that $t s \geq x$.

LEMMA 4.4. For any execution $\xi o f S$, if a postit $=x$ is introduced in the system by a read operation $\rho_{1}$, then any subsequent read operation will observe a maximum timestamp $t s^{\prime}$ such that $t s^{\prime} \geq x$.

PROOF. Consider an execution $\xi$ of SF where the read operation $\rho_{1}$ introduced a postit equal to $y$ to the system. It follows that $\rho_{1}$ observed as the maximum timestamp in the system $T S_{1}=x$. As $\left|M S_{1}\right| \geq S-\alpha t$ and $\mid \cap_{m \in M S_{1}}$ m.seen $\mid=\alpha, \rho_{1}$ performs an informative operation. Since $\alpha \in[1, V+1]$ and $S>(V+$ 2)t, we get that $\left|M S_{1}\right|>t$. So, if we denote by $S_{2}$ the set of servers that replied to a subsequent $\operatorname{read} \rho_{2}\left(\left|S_{2}\right|=S-t\right)$, then per Lemma 4.2 there is a server, $s_{i} \in \operatorname{Max}_{1} \cap S 2$ that replies to $\rho_{2}$ with a timestamp $t s^{\prime} \geq x$. Therefore, $\rho_{2}$ will detect a maximum timestamp $T S_{2} \geq t s^{\prime}$, and hence $T S_{2} \geq x$.

LEMMA 4.5. For any execution $\xi$ of $S F$ if a read operation $\rho_{1}$ receives a postit $=x$ then $\rho_{1}$ will return a value $y \geq x$.

Proof. Consider an execution $\xi$ of SFwhich contains a read operation $\rho_{1}$ by a reader $\left\langle r_{i}, \nu_{i}\right\rangle$. It follows from Lemma 4.4 that if read $\rho_{1}$ receives a postit $=x$, then it will detect a maximum timestamp $T S_{1} \geq x$. Let $T S_{1}=x$ and so either the predicate will hold and then $\rho_{1}$ will return $y=T S_{1}$, or the condition whether postit $_{r_{i}}=T S_{1}$ will be true and so $\rho_{1}$ will in this case return 
$y=T S_{1}$ as well. Thus $\rho_{1}$ will return $y=x$. If now $T S_{1}>x$ then $\rho_{1}$ will return $y=T S_{1}$ if the predicate holds or $y=T S_{1}-1$ otherwise. Note that since postit $=x$, it is less than $T S_{1}$ and so the postit condition does not hold. Either case $\rho_{1}$ will return a value $y \geq x$.

The following lemma ensures the second atomicity property.

LEMMA 4.6. For any execution $\xi$ of $S F$, if a read $\rho_{1}$ is complete and succeeds some write $(k)$, then $\rho_{1}$ returns $\ell$ such that $\ell \geq k$.

PROOF. Suppose that the writer $w$ performs a write $(k)$ operation and precedes the read $\rho_{1}$ operation by reader $r_{i}$ with virtual id $\nu_{i}$ during an execution $\xi$ of SF. Let $S_{w}$ be the $S-t$ servers that replied to $w$ in the same execution. The intersection between $S_{w}$ and $S_{1}, \operatorname{Max} S_{1}=S_{w} \cap S_{1}$, is obviously $\left|\operatorname{Max} S_{1}\right| \geq S-2 t$. Since $w r$ preceded $\rho_{1}$ the timestamp $t s$ for each server in $\operatorname{Max} S_{1}$, per Lemma 4.2 it is greater or equal to $k$. So $\rho_{1}$ received a maximum timestamp $T S_{1}$ such that $T S_{1} \geq k$. From the implementation we know that the reader returns either $T S_{1}$ or $T S_{1}-1$. We consider two cases:

Case 1: $T S_{1}>k$. Since $\rho_{1}$ returns either $T S_{1}$ or $T S_{1}-1$, it follows that either case it returns a timestamp greater or equal to $k$. Case 2: $T S_{1}=k$. As we mentioned above each server in $\operatorname{Max}_{1}$ replies with a $t s \geq k$. Since $T S_{1}=k$ every server $s_{i} \in \operatorname{Max} S_{1}$ replies with a timestamp $t s=k$ to $\rho_{1}$. So the set $M S_{1}$, which contains the messages received by $\rho_{1}$ with the highest timestamp, will include the messages sent by all the servers in $\operatorname{Max} S_{1}$. So $|M S| \geq S-2 t$. But since the writer sent a message with timestamp $k$ to the servers before $\rho_{1}$, then $w$ is included in the seen set of each server in $\operatorname{Max} S_{1}$. Before the servers in $M a x S_{1}$ responded to $\rho_{1}$ they also included $\nu_{i}$ in their seen set. So the predicate will be true for $\alpha=2$ and $\rho_{1}$ will return $T S_{1}=k$. Observe that no reader will return $T S_{1}$ because of a postit in the system because the predicate will hold for every process in the system for $\alpha=2$, since the writer $w$ has no sibling processes.

In order to prove the forth atomicity property, we first need to show that readers who belong to the same virtual node (siblings) satisfy that property. Then we show that the property is also true for any two non-sibling readers in the system.

LEMMA 4.7. Let the readers $\left\langle r_{j}, \nu_{k}\right\rangle$ and $\left\langle r_{i}, \nu_{k}\right\rangle$ be siblings and perform the read operations $\rho_{1}$ and $\rho_{2}$ respectively. For any execution $\xi$ of $S F$ that contains $\rho_{1}$ and $\rho_{2}$, if $\rho_{1}$ precedes $\rho_{2}$, and $\rho_{1}$ returns $x$ then $\rho_{2}$ returns $y$, such that $y \geq x$.

PROOF. Consider an execution $\xi$ of SF. Let first investigate the case where $r_{j}=r_{i}$. In this case $\rho_{1}$ denotes the first read operation of $r_{j}$ and $\rho_{2}$ a succeeding read operation from the same reader. Let $x$ be the value returned from $\rho_{1}$. During the read $\rho_{2}, r_{j}$ sends a READ message with $t s_{r_{j}}=T S_{1} \geq x$. This message will be received by all servers in $S_{2}$ which according to Lemma 4.2 will reply with a timestamp $t s^{\prime} \geq T S_{1} \geq x$. So $T S_{2} \geq x$. If $T S_{2}=x$ then $\left|M S_{2}\right|=S-t$ and the predicate holds for $\alpha=1$. Thus $y=T S_{2}=x$. Otherwise, if $T S_{2}>x$, the return value $y$ will be equal to $T S_{2}$ or $T S_{2}-1$ and thus $y \geq x$. By a simple induction we can show that this is true for every read operation of $r_{j}$ (including $\rho_{2}$ ) after $\rho_{1}$. For the rest of the proof we assume that $r_{j} \neq r_{i}$. We investigate the following two possible cases: (1) $\rho_{1}$ returns $x=$ $T S_{1}-1$ and (2) $\rho_{1}$ returns $x=T S_{1}$. In all of the cases we show that $x \leq y$ or that the case is impossible.

Case 1:In this case $x=T S_{1}-1$. Therefore, some servers replied to $\rho_{1}$ with $T S_{1}=x+1$, and hence a write $(x+1)$ operation had started before $\rho_{1}$ is completed. So write $(x)$ completed before $\rho_{1}$ has completed and moreover before $\rho_{2}$ is executed since $\rho_{1}$ precedes $\rho_{2}$. Thus by Lemma $4.6 \rho_{2}$ returns a value $y \geq x$.

Case 2:In this case $x=T S_{1}$. Hence either there is some $\alpha \in[1, V+1]$ such that $\left|M S_{1}\right| \geq S-\alpha t$ and $\mid \cap_{m \in M S_{1}}$ m.seen $\mid \geq \alpha$ or $\rho_{1}$ received a postit equal to $T S_{1}$ from some server. We examine those two possibilities separately.

Case 2(a): It follows that $x=T S_{1}$, and there is some $\alpha \in[1, V+1]$ such that $M S_{1}$ consist at least $S-\alpha t$ messages received by $\rho_{1}$ with $t s=x$ and $\mid \cap_{m \in M S_{1}}$ m.seen $\mid \geq \alpha$. Since $V<\frac{S}{t}-2$ and $a \in[1, V+1]$, then $\left|M S_{1}\right|=S-a t>t$. We have two cases to consider for $\rho_{1}$ : (1) First let examine the case where $\rho_{1}$ returns $x=T S_{1}$ because $\mid \cap_{m \in M S_{1}}$ m.seen $\mid=\alpha$. According to the implementation, $\rho_{1}$ has to inform $\left|N S_{1}\right| \geq 2 t+1$ servers about its return value, $x$. Since $\rho_{1}$ precedes $\rho_{2}$, at least $\left|N S_{1} \cap S_{2}\right| \geq 2 t+1$ servers, that informed by $\rho_{1}$, will reply to $\rho_{2}$. Any server $s_{i} \in N S_{1} \cap S_{2}$, by Lemma 4.4 will reply with a postit $\geq x$ to $\rho_{2}$ and with a timestamp $t s \geq x$. So $\rho_{2}$ will observe a maximum timestamp $T S_{2} \geq x$. According now to Lemma 4.5 $\rho_{2}$ will return a value $y \geq x$. (2) The second case arise when $\rho_{1}$ returns $x=T S_{1}$ because $\mid \cap_{m \in M S_{1}}$ m.seen $\mid>\alpha$. We can split this case in two subcases regarding the value returned by $\rho_{2}$. The two possible values that $\rho_{2}$ might return is $y=T S_{2}$ or $y=T S_{2}-1$ :

(i) Let first consider the case where $y=T S_{2}$. Since $\rho_{1}$ returned $x=T S_{1}$, as we mentioned in (1), there is a write $(x)$ operation that preceded or was concurrent with $\rho_{1}$. As stated above $\left|M S_{1}\right|>t$ and hence there is a server $s_{i}$ such that $s_{i} \in \operatorname{Max} S_{1} \cap S_{2}$. By Lemma 4.2, $s_{i}$ will send a timestamp $t s \geq x$ to $\rho_{2}$, and hence $T S_{2} \geq t s$. So $y \geq x$.

(ii)We now get down to the case where $\rho_{2}$ returns $y=T S_{2}-1$. Since $\left|M a x S_{1}\right|>t$, there must be a server $s_{i} \in \operatorname{Max} S_{1} \cap S_{2}$ and $s_{i}$ replies with a timestamp $t s \geq x$ to $\rho_{2}$. So the highest timestamp in $S_{2}$ (i.e. $T S_{2}=y+1$ ) will be greater or equal to $x$. If the inequality is true, namely $y+1>x$, then clearly the value returned by $\rho_{2}$ is $y \geq x$. If the equality holds and $y+1=x$ then the highest timestamp received by $\rho_{2}, T S_{2}=y+1=x$. Hence all the servers in $\operatorname{Max} S_{1} \cap S_{2}$ replied with a timestamp $t s=x=y+1$ to $\rho_{2}$. Recall that this case arise only when $\mid \cap_{m \in M S_{1}}$ m.seen $\mid>\alpha$. Also according to Lemma 4.1, ||$M S_{2}|-| M S_{1}|| \leq t$ and hence $\left|M S_{2}\right| \geq S-(\alpha+1) t$. For any $s_{i} \in \operatorname{Max} S_{1} \cap S_{2}$, we denote as $m_{1}$ the message sent by $s_{i}$ to $\rho_{1}$ and $m_{2}$ the message sent to $\rho_{2}$. Obviously $m_{1}$.ts $=m_{2}$.ts $=x$. Since the timestamp is the same and $m_{1}$ sent before $m_{2}$ then $m_{1}$.seen $\subseteq m_{2}$.seen. As a result $\mid \cap_{m \in M S_{1}}$ m.seen $|\leq| \cap_{m \in M S_{2}}$ m.seen $\mid$. Notice that, since the two readers are siblings, if no non-sibling reader received replies from those servers in between $\rho_{1}$ and $\rho_{2}$, then $m_{1}$.seen $=m_{2}$.seen and $\mid \cap_{m \in M S_{1}}$ m.seen $|=| \cap_{m \in M S_{2}}$ m.seen $\mid$. Either case, $\mid \cap_{m \in M S_{2}}$ m.seen $\mid>\alpha$ and hence $\mid \cap_{m \in M S_{2}}$ m.seen $\mid \geq \alpha+1$. Observe that the predicate now is true for $\alpha+1$ since $\left|M S_{2}\right| \geq S-(\alpha+1) t$, and thus $\rho_{2}$ must return $T S_{2}=x=y+1$, contradicting the initial assumption that $y=x+1$. The same result applies in both cases where $\alpha \leq V$ and $\alpha=V+1$ since the seen set remains unchanged.

Case 2(b):Here $\rho_{1}$ returns $x=T S_{1}$ because there was not $\alpha \in[1, \ldots, V+1]$, such that $\mid \cap_{m \in M S_{1}}$ m.seen $\mid \geq \alpha$, but some postits equal to $T S_{1}$ received by $\rho_{1}$. We have to consider 2 cases here. Either (1) $\rho_{1}$ received more than $t+1$ postits, or (2) $\rho_{1}$ received less than $t+1$ postits. Both cases imply that, a reader $\left\langle r_{m}, \nu_{n}\right\rangle$ perform a read $\rho_{1 a}$, and is about to return or already 
returned the maximum timestamp(which is equal to $T S_{1}$ ) in the system. Furthermore implies that $\rho_{1 a}$ initiated an informative phase which is concurrent or precedes the read operation $\rho_{1}$. By analyzing the cases we obtain the following results:

(1) If $\rho_{1}$ received more than or equal to $t+1$ messages containing a postit with value postit $=T S_{1}=x$, then the writer $w$ initiated a write $(x)$ operation during or before $\rho_{1}$ completed. It follows that $N S_{1 a} \cap S_{1}$ denote the set of servers that replied to $\rho_{1}$ and contained the postit $=T S_{1}$. The reader $\rho_{2}$ receives replies from $\left|S_{2}\right|=S-t$ servers. Since $\left|N S_{1 a} \cap S_{1}\right| \geq t+1$, then $\left|S 2 \cap\left(N S_{1 a} \cap S_{1}\right)\right| \geq 1$. So the read operation $\rho_{2}$ will receive a reply from at least one server $s_{i} \in N S_{1 a} \cap S_{1}$. Hence, from Lemma 4.3, $\rho_{2}$ receives a postit $\geq x$ from $s_{i}$ and according to Lemma 4.5 will return a value $y \geq$ postit and thus $y \geq x$.

(2) Let now examine if $\rho_{1}$ received less than $t+1$ messages containing postits with value equal to $T S_{1}$. Let assume again that $\left|N S_{1 a} \cap S_{1}\right|<t+1$ is the set of servers that replied with postit $=T S_{1}$ to $\rho_{1}$. However, in contrary to the previous case, the situation where $\left|\left(N S_{1 a} \cap S_{1}\right) \cap S 2\right|=0$ might arise. So $r_{i}$ gets into the information process in order to inform sufficient servers about its potential return timestamp. So at the time where $\rho_{1}$ is completed, $\left|N S_{1}\right| \geq 2 t+1$ servers contain a postit $\geq T S_{1}$. When $\rho_{2}$ is performed, $T S_{2}$ is greater than or equal to $x$, since there is a server $s_{i} \in M S_{1} \cap S_{2}$ and, according to Lemma 4.4, $s_{i}$ returns a timestamp $t s \geq x$. Furthermore there is a server $s_{j} \in N S_{1} \cap S_{2}$, and so according to Lemma $4.3, s_{j}$ replies with a postit $\geq x$. So, by Lemma $4.5, \rho_{2}$ returns a value $y \geq x$.

Similarly we proof that the fourth atomicity properties is also satisfied for any two non-sibling reader processes in the system.

LEMMA 4.8. Let the readers $\left\langle r_{j}, \nu_{j}\right\rangle$ and $\left\langle r_{i}, \nu_{i}\right\rangle$ be nonsiblings and perform the read operations $\rho_{1}$ and $\rho_{2}$ respectively. For any execution $\xi$ of $S F$ that contains $\rho_{1}$ and $\rho_{2}$, if $\rho_{1}$ precedes $\rho_{2}$, and $\rho_{1}$ returns $x$ then $\rho_{2}$ returns $y$, such that $y \geq x$.

THEOREM 4.9. Algorithm SF implements an atomic read/write register in the SWMR model.

PROOF. It follows from the fact that every process guarantees termination by waiting for only $S-t$ replies and the lemmas proved above.

\section{IMPOSSIBILITY}

As it is shown in [3], no fast implementations exist if the number of readers $R$ in the system is such that $R \geq \frac{S}{t}-2$. Our approach to semifast solutions is to trade fast implementation for increased number of readers, while enabling some (many) reads to be fast. Here we show that semifast implementations are possible if and only if the number of virtual identifiers (virtual nodes) in the system is less than $\frac{S}{t}-2$. We show that the bound on the virtual identifiers is tight for algorithms that: (1) do not use any grouping assumptions and thus consider each node acting individually in the system, and (2) consider grouping mechanisms such as in algorithm SF. In our context by "grouping mechanism" we only mean the grouping of the reader processes in any arbitrary fashion. In other words we omit the grouping techniques that involve grouping of non-reader processes in the system. Additionally, Lemma 5.2, shows that informing at least $3 t+1$ servers during a second communication round is a tight bound for any semifast implementation.

In algorithms where there is no grouping mechanisms assumed we can consider each reader to form an individual group. So the number of virtual nodes $V$ is equal to the number of readers $R$. As showed in [3] in such systems there is no fast implementation of the $\mathrm{read} /$ write register if $R \geq \frac{S}{t}-2$. However this violates the fourth property of the semifast definition and thus no such systems can be semifast. Hence our bound applies in these kind of systems. We now show the following considering algorithms using a grouping mechanism similar to SF:

LEMMA 5.1. No semifast implementation exists if the number of node groups $V$ in the system is $\geq \frac{S}{t}-2$.

The following lemma shows that the existence of a semifast implementation also depends on the number of minimum messages sent by a process during its second communication round.

LEMMA 5.2. There is no semifast implementation of an atomic register if a read operation informs $3 t$ or fewer servers during its second communication round.

We now state the main result of this section.

THEOREM 5.3. No semifast implementation I exists if the number of virtual nodes in the system is $\geq \frac{S}{t}-2$ and if $3 t$ or fewer servers are informed during a second communication round.

PROOF. It follows directly from Lemmas 5.1 and 5.2.

\section{MWMR MODEL}

In this section we consider the multiple writer - multiple reader (MWMR) model and show that no semifast implementations of atomic registers are possible in this setting in the presence of server failures.

Preliminaries. For the MWMR model we relax the definition of a semifast implementation as presented for the SWMR model, by allowing read operations to perform more than two communication rounds (i.e., instead of two rounds we allow multiple rounds in Definition 2.2). First we extract several immediate properties from the definition of atomicity presented in Section 2. If for given operations $\pi_{1}$ and $\pi_{2}$ in an execution, the response step of $\pi_{1}$ precedes the invocation step of $\pi_{2}$, we denote this by $\pi_{1} \rightarrow \pi_{2}$. To satisfy the atomicity definition the following properties must be true for any execution of the MWMR semifast implementation. PROPERTY P1: if there is a write operation $w r$ that writes value $v$ and a read operation $\rho_{i}$ such that $w r \rightarrow \rho_{i}$, and all other writes precede $w r$ then $\rho_{i}$ returns $v$. PROPERTY P2: if the response steps of all write operations precede the invocation steps of the read operations $\rho_{i}$ and $\rho_{j}, i \neq j$, then $\rho_{i}$ and $\rho_{j}$ must return the same value. PROPERTY P3: If the response steps of all the write operations precede the invocation step of a read operation $\rho_{i}$ then $\rho_{i}$ returns a value written by some complete write.

For the reasons discussed in Section 2, we assume the communication scheme where a server replies to a READ (or WRITE or INFORM) message without waiting to receive any other READ (or WRITE or INFORM) messages. In this proof we say that an operation performs a read phase during a communication round if it gathers information from the system at that round. We say that an operation performs a write phase during a communication round if it propagates information to other participants at that round. A read phase of an operation (read or write) does not modify the value of the atomic object. On the other hand a write phase of an operation $\pi$ behaves as follows according to its type: (1) a new, currently unknown value is written to the register, if $\pi$ is a write operation (2) only previously known values are written to the register if $\pi$ is a read operation.

We say that a complete operation $\pi$ skips a server $s_{i}$ if $s_{i}$ does not receive any messages from the process $p$ that invoked $\pi$ and and 
the process $p$ does not receive any replies from $s_{i}$. All other servers that receive the READ, WRITE or INFORM messages from $p$ reply to these, and $p$ receives these replies. All other messages remain in transit. Since we assume that $t=1$, any complete operation may skip at most one server. We say that an operation is skip-free if it does not skip any server.

Since we consider read operations that might perform multiple communication rounds to complete, we denote by $r_{i}(j)$ the $j^{\text {th }}$ communication round of a read operation from reader $r_{i}$. An arbitrary delay may occur between two communication rounds $r_{i}(j)$ and $r_{i}(j+1)$ where other read (write) operations or read (write) phases might be executed. So we define as $s r_{i}(j-1)$ a set of operation phases (read or write) with the property that any $\pi \in s r_{i}(j-1), \pi \rightarrow r_{i}(j)$. A set $s r_{i}(j-1)$ might be equal to the empty set containing no operations.

CLAIM 6.1. A read operation $\rho$ that succeeds any write operation $\omega$ or write phase $\omega p$ of an operation $\pi \neq \rho$, returns the value decided by the read phase preceding its last write phase.

Construction and Main Result. We now present the construction we use to prove the main result. We show execution constructions assuming that two writers $\left(w_{1}\right.$ and $\left.w_{2}\right)$, and two readers $\left(r_{1}\right.$ and $\left.r_{2}\right)$ participate in the system. We assume skip-free operations since they comprise the best case scenario and thus a lower bound for these is sufficient. Let us first consider the finite execution fragment $\varphi_{1}$, constructed from the following skipfree, complete operations: (a) operation write (2) by $w_{2}$, (b) operation write(1) by $w_{1}$, and (c) operation read re $_{1}$ ) by $r_{1}$. These operations are not concurrent and they are executed in the order write $(2) \rightarrow$ write $(1) \rightarrow \operatorname{read}_{1}()$. By property $\mathrm{P} 2$, operation $\operatorname{read}_{1}()$ returns 1.

We now invert the write operations of the above execution and we obtain execution $\varphi_{2}$, consisting of the following skip-free, complete operations in the following order: (a) operation write(1) by $w_{1}$, (b) operation write (2) by $w_{2}$, and (c) operation $\operatorname{read}_{1}($ ) by $r_{1}$. As before, these operations are not concurrent. So in this case, by property $\mathrm{P} 2$, operation read $_{1}()$ returns 2 .

The generalization $\varphi_{1 g}$ of $\varphi_{1}$, for $1 \leq i \leq n$, when the reader $r_{1}$ performs $n$ communication rounds is the following: (a) a write (2) operation from $w_{2}$, (b) a write(1) operation from $w_{1}$, (c) a set of read operations $s r_{1}(i-1)$ from readers $r_{j}, j \neq 1$, and (d) a read or a write phase $r_{1}(i)$ of the read r $_{1}$ operation from reader $r_{1}$. Notice that for $n=1$ and for $s r_{1}(0)=\emptyset$ no process can distinguish $\varphi_{1 g}$ from $\varphi_{1}$. Clearly at the end of the $n^{t h}$ communication round, by property $\mathrm{P} 2$, the operation $\mathrm{read}_{1}()$ from $r_{1}$ returns 1 .

Similarly we define the $\varphi_{2 g}$ to be the generalization of $\varphi_{2}$, where the write operations are inversed: (a) a write(1) operation from $w_{1}$, (b) a write (2) operation from $w_{2}$, (c) a set of read operations $s r_{1}(i-1)$ from readers $r_{j}, j \neq 1$, and (d) a read or a write phase $r_{1}(i)$ of the $\operatorname{read}_{1}()$ operation from reader $r_{1}$. In this case by the end of the $n^{\text {th }}$ communication round of $r_{1}$, and by property $\mathrm{P} 2$, the $\operatorname{read}_{1}()$ operation returns 2 .

If we assume now, without loss of generality, that the last communication round $r_{1}(n)$ of $r_{1}$ in $\varphi_{1 g}$ is a write phase, then $r_{1}$ should not be able to differentiate $\varphi_{1 g}$ from the following execution, for $1 \leq i \leq n-1$ : (a) a write (2) operation from $w_{2}$, (b) a write (1) operation from $w_{1}$, (c) a set of read operations $s r_{1}(i-1)$ from readers $r_{j}, j \neq 1$, (d) a read phase $r_{1}(i)$ of the $\operatorname{read}_{1}()$ operation from reader $r_{1}$, (e) a set of read operations $s r_{1}(n-1)$ from readers $r_{j}, j \neq 1$, and (f) a write (1) operation from $r_{1}(n)$. By operation write (1), the reader $r_{1}$ tries to disseminate the information gathered from the previous rounds regarding the value of the atomic object. Similarly we can define $\varphi_{2 g}$ with the difference that reader $r_{1}$ will perform a write(2) operations during its last communication round.

Obviously we have the same setting as in Claim 6.1 and so by the same claim the decision for the return value must be made in $r_{1}(n-1)$. Notice that the decision of $r_{1}$ taken in $r_{1}(n-1)$ is not affected from the operations in $\operatorname{sr}(n-1)$. So we can assume that $\varphi_{1 g}$ and $\varphi_{2 g}$ contain only read phases by $r_{1}$. According now to property $\mathrm{P} 2, r_{1}$ will return 1 by the end of $r_{1}(n-1)$ in $\varphi_{1 g}$ and 2 by the end of $r_{1}(n-1)$ in $\varphi_{2 g}$. Since we assume that we only have 2 readers in the system $r_{1}$ and $r_{2}$ and since $r_{2}$ does not perform any read operation in either $\varphi_{1 g}$ or $\varphi_{2 g}$, we have that all the sets $s r_{1}(i-1)=\emptyset$ for $1 \leq i \leq n$ in both executions $\varphi_{1 g}$ and $\varphi_{2 g}$.

THEOREM 6.2. If the number of writers in the system is $W \geq$ 2 , the number of readers is $R \geq 2$, and $t \geq 1$ servers may fail, then there is no semifast atomic register implementation.

PROOF. The proof follows by reasoning on the construction presented above. See [5] for full details.

\section{SIMULATION RESULTS}

To evaluate the effectiveness of our implementation, we simulated algortihm $S F$ using the NS2 network simulator and measured the percentage of two-round read operations as a function of the number of readers and the number of faulty servers. The testbed of our simulations included 20 servers out of which 5 may fail at arbitrary times. Since we require that $V<\frac{S}{t}-2$, in order to maintain at least one group we can tolerate up to $\frac{S}{4}$ faulty servers. The number of reader processes varies between 10 and 80 . We use $r$ Int and wInt to stand for the time intervals between each read and write operations respectively. Several scenarios were tested: (i) frequent reads and infrequent writes, where $r$ Int $<w$ Int, $(i i)$ concurrent reads and writes, such that $r$ Int $=w$ Int, and $(i i i)$ infrequent reads and frequent writes, such that $r$ Int $>w$ Int. The processes send their messages after a random delay to model asynchrony. According to our setting only the messages between the invoking processes and the servers, and the replies from the servers are delivered (no messages are exchanged between any servers or among the invoking processes).

Stochastic simulations. This is the class of executions where each read (resp. write) operation from an invoking process is scheduled at random time between $1 \mathrm{sec}$ and $r$ Int (resp. wInt) after the last read (resp. write) operation. Introducing randomness in the operation invocations renders a more realistic scenario where processes are interacting with the atomic object independently. Under this setting, for the three scenarios $(i),(i i)$, and (iii), the comparisons between $r$ Int and $w$ Int may be satisfied only stochastically. A single value of $w$ Int $=4.3 \mathrm{sec}$ was chosen for the upper limit of any write operation. For the read operations the values of $r$ Int $=2.3 \mathrm{sec}, r$ Int $=4.3 \mathrm{sec}$, and $r$ Int $=6.3 \mathrm{sec}$ were chosen, with the results presented in Figure 2, set a. The results for this family of executions are similar where the percentage of tworound reads is mainly affected by the number of faulty servers. In all cases the percentage of two-round reads is under $7.5 \%$.

Fixed interval simulations. Here the intervals for each read (or write) operation are fixed at the beginning of the simulation. All readers use the same interval $r$ Int, and the writer the interval $w$ Int. This family of simulations represent conditions where operations can be frequent and bursty. The intervals $r$ Int and $w$ Int when $r$ Int $\neq w$ Int are chosen to avoid having read operations invoked at the same time with write operations. In Figure 2, b(i) illustrates the case of $r$ Int $<w$ Int. A read (write) operation is invoked by every reader (resp. writer) in the system every $r$ Int $=2.3 \mathrm{sec}$ 


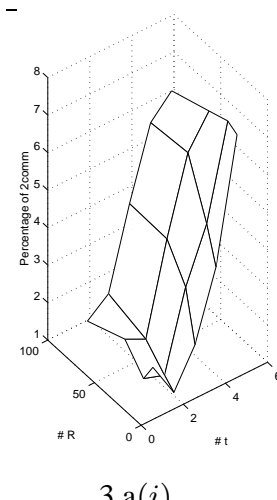

3. $\mathrm{a}(i)$

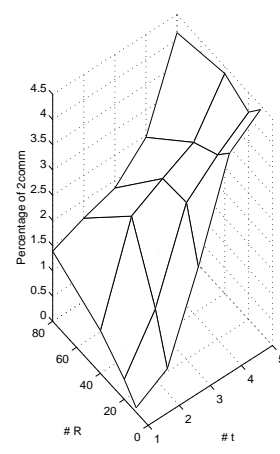

3.b $(i)$

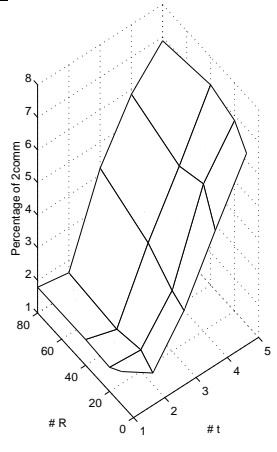

3.a $(i i)$

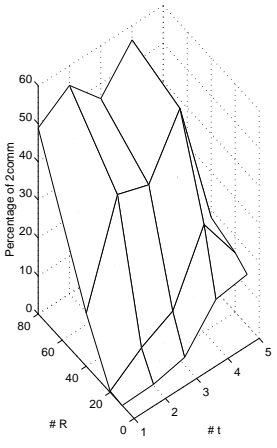

3.b $(i i)$
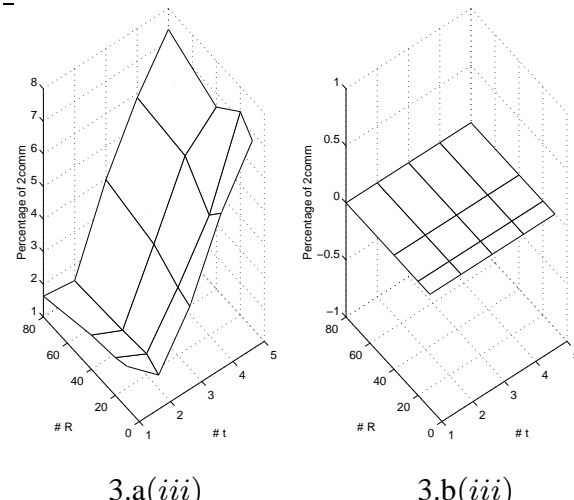

3.b $($ iii $)$

Figure 2: Stochastic simulation 3.a; Fixed interval simulation 3.b. The vertical axes show the percentage of two-round reads as a function of the number of readers and the number of faulty servers.

(resp. $w I n t=4.3 \mathrm{sec}$ ). Because of asynchrony not every read operation completes before the invocation of the write operation and thus we observe a small percentage of reads that perform two communication rounds. In $\mathrm{b}$ (ii) the condition where $r$ Int $=w$ Int is illustrated. This is the worst case scenario since all operations, read or write, are invoked at the same time, that is they are invoked every $r$ Int $=w I n t=4.3 \mathrm{sec}$. Although the conditions in this case are highly adversarial, we observe that only about half of the read operations perform two communication rounds. Lastly, in b(iii) we study the case where $w$ Int $<$ rInt. In particular a read operation is invoked every $r$ Int $=6.3 \mathrm{sec}$ by each reader and a write operation every $w$ Int $=4.3 \mathrm{sec}$. In this case all write operations complete before any invocation step of a read operation. So all the servers reply to any read operation with the latest timestamp and thus no read operation needs to perform a second communication round. Finally, note the common trend that increasing the number of readers and the number of faulty servers negatively impacts the performance of the algorithm in the first two scenarios.

\section{CONCLUSIONS AND FUTURE WORK}

In this paper we investigated the existence of semifast implementations of a read/write atomic register. It is shown in [3] that there are no fast SWMR implementations-where both readers and the writer perform one communication round-if there are $\frac{S}{t}-2$ or more readers. Furthermore a question was posed whether there exist semifast implementations where reads or writes are fast.

The goal of this paper is to relax the bound on the readers in the system at the cost of allowing some reads to perform two communication rounds. We formalized the notion of semifast implementations and we presented an implementation that meets our goal and satisfies the required properties. For our implementation we show that between two write operations only one complete read operation needs to perform two communication rounds. We also showed that there is no semifast implementation if the number of different virtual nodes in the system is $\frac{S}{t}-2$ or greater. Moreover we showed that there cannot exist semifast implementations for the MWMR model. Finally, we simulated our algorithm and presented the results that demonstrate that most read operations are fast in our simulated executions.

Our paper made progress in identifying the tradeoffs between the concurency in the system and the number of communication rounds required to implement atomic registers. The next step is to better understand the tradeoffs in the MWMR model. One direction is to consider hybrid semifast implementations where writers and readers perform a mixture of fast and semifast operations. Another direction is to consider dynamic settings such as [7] where nodes might join, leave and arbitrarily fail. The broader question we intend to investigate is-given a particular distributed system model-how fast can a distributed atomic read be?

Acknowledgment: We thank Rachid Guerraoui for his helpful comments.

\section{REFERENCES}

[1] H. Attiya, A. Bar-Noy, and D. Dolev. Sharing memory robustly in message passing systems. $J$. of the ACM, 42(1):124-142, 1996.

[2] S. Dolev, S. Gilbert, N. Lynch, A. Shvartsman, and J. Welch. Geoquorums: Implementing atomic memory in mobile ad hoc networks, 2003.

[3] P. Dutta, R. Guerraoui, R. R. Levy, and A. Chakraborty. How fast can a distributed atomic read be? In Proceedings of the twenty-third annual ACM symposium on Principles of distributed computing, pages 236-245. ACM Press, 2004.

[4] B. Englert and A. A. Shvartsman. Graceful quorum reconfiguration in a robust emulation of shared memory. In International Conference on Distributed Computing Systems, pages 454-463, 2000.

[5] C. Georgiou, N. Nicolaou, and A. Shvartsman. Fault-tolerant semifast implementations for atomic read/write registers, 2005. http://www.cse.uconn.edu/ ncn03001/pubs/TRs/GNS06.pdf.

[6] N. Lynch. Distributed Algorithms. Morgan Kaufmann Publishers, 1996.

[7] N. Lynch and A. Shvartsman. RAMBO: A reconfigurable atomic memory service for dynamic networks. In Proc. of 16th International Symposium on Distributed Computing, pages 173-190, 2002.

[8] N. A. Lynch and A. A. Shvartsman. Robust emulation of shared memory using dynamic quorum-acknowledged broadcasts. In Symposium on Fault-Tolerant Computing, pages 272-281, 1997.

[9] P. Vitanyi and B. Awerbuch. Atomic shared register access by asynchronous hardware. In 27th Annual IEEE Symposium on Foundations of Computer Science, pages 233-243, 1986. 